\title{
Nodal Solutions for a Sublinear Elliptic Equation
}

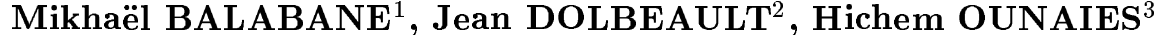

1 Département de Mathématiques, Institut Galilée, Université Paris 13, 99, avenue JeanBaptiste Clément, 93430 Villetaneuse, France - E-mail: balabane@math.univ-paris13.fr

2 Ceremade, U.M.R. C.N.R.S. no. 7534, Univ. Paris IX-Dauphine, Pl. de Lattre de Tassigny, 75775 Paris Cédex 16, France - E-mail: dolbeaul@ceremade.dauphine.fr

3 Département de Mathématiques, Faculté des sciences de Monastir, Route de l'environnement, 5019 Monastir, Tunisia - E-mail: Hichem.Ounaies@fsm.rnu.tn

March 22, 2001

Abstract: We consider radial solutions of $\Delta u+u-|u|^{-2 \theta} u=0$ in $\mathbb{R}^{d}$ with $d>1, \theta \in\left(0, \frac{1}{2}\right)$ and prove by a shooting method the existence of compactly supported solutions with any given number of nodes.

\section{Introduction and main result}

We consider radial solutions in $\mathbb{R}^{d}$ of

$$
\Delta u+f(u)=0
$$

where $f(u)=u-|u|^{-2 \theta} u$ with $\theta \in\left(0, \frac{1}{2}\right)$. If we put $r=|x|$ and denote by ' the derivative w.r.t. $r$, any solution $u(x) \equiv u(r)$ shows a $C^{2}$ regularity and fulfills the O.D.E.

$$
\left\{\begin{array}{l}
u^{\prime \prime}+\frac{d-1}{r} u^{\prime}+f(u)=0 \\
u(0)=\lambda, \quad u^{\prime}(0)=0
\end{array}\right.
$$

for some $\lambda \in \mathbb{R}$. For $d>1$, we prove that solutions of (2) globally exist for any $\lambda \in \mathbb{R}$, are bounded and have a finite number of nodes, i.e. of roots inside the interior of their support. We also prove that those which are in $H^{1}\left(\mathbb{R}^{d}\right)$ (or equivalently tend to 0 as $r \rightarrow+\infty$ ) turn out to be compactly supported (other solutions converge to \pm 1 as $r \rightarrow+\infty$ ). Our main result is the following.

Theorem 1. Let $d>1$. There exists an unbounded increasing sequence $\left(\lambda_{k}\right)_{k \in \mathbb{N}}$ of initial data such that for any $k \in \mathbb{N}$, (1) has a radial compactly supported solution in $H^{1}\left(\mathbb{R}^{d}\right)$ with exactly $k$ nodes, corresponding to $\lambda=\lambda_{k}$.

One of the main motivations for the analysis of spherically symmetric (or radial) solutions of $\Delta u+\omega^{2} u+g(u)=0$ arises from the theory of standing waves $v(x, t)=e^{i \omega t} u(x)$ for the semilinear wave equation

$$
v_{t t}-\Delta v=g(v),
$$


with finite energy $\int_{\mathbb{R}^{d}}\left(\frac{1}{2}\left|v_{t}\right|^{2}+\frac{1}{2}|\nabla v|^{2}-G(v)\right) d x$, where $G(v)=\int_{0}^{v} g(s) d s$. Here $v$ is complex valued and we assume that $g\left(\varrho e^{i \varphi}\right)=e^{i \varphi} g(\varrho)$ for any $\varrho, \varphi \in \mathbb{R}$. Such solutions (and especially the radial ones) have been extensively studied (see [6, $12,19,23,25,28])$ for Lipschitz nonlinearities. When $g(u)=-|u|^{-2 \theta} u, \theta \in\left(0, \frac{1}{2}\right)$, the non Lipschitz character of the nonlinearity at $u=0$ implies that solutions in $H^{1}\left(\mathbb{R}^{d}\right)$ are localized, not only in the sense that $u(x) \rightarrow 0$ as $|x| \rightarrow+\infty$, but that they are actually compactly supported in $\mathbb{R}^{d}$ (see $[11,27]$ ). Note that for power nonlinearities, $\omega$ can always be taken equal to 1 up to a scaling (the equation for $x \mapsto \omega^{1 / \theta} u\left(\omega^{-1} x\right)$ is Equation (1)).

Equation (1) also appears in various other problems, for instance as the large time limit of the semilinear heat equation $u_{t}-\Delta u=f(u)$ (see [9] for a related problem) and shows up in several chemical reactions or plasma physics models (see $[13,22,11])$.

For many types of nonlinearities, variational methods have proved their efficiency in getting multiplicity results (see $[28,6])$. The fact that the solutions are ordered by their number of roots has been proved in a regular framework using an approach based on dynamical systems methods and estimates of the winding number by C. Jones \& T. Küpper in [19] (see references therein for earlier partial results). This has been done in the framework of a $C^{1}$ superlinear and subcritical nonlinearity, and extended by several methods in $[12,23,17,20]$ to various but still Lipschitz superlinear and subcritical nonlinearities (also see [20] in the case of $u \log u$ ). We shall refer to the references quoted in these papers for more details on the superlinear case, concerning, for instance, the uniqueness of the ground state solution.

Dynamical systems methods for nonautonomous equations have been used to get results on the nodes in many other contexts, for instance in the case of the Dirac equation with a smooth $[1,2]$ or even non Lipschitz at the origin [3] nonlinearity and it is certainly out of the scope of this paper to give a complete list of references.

When the nonlinearity is globally Lipschitz, solutions with a prescribed number of nodes have an infinite support, which makes the counting of the nodes uneasy. Such a difficulty can in some cases be solved by an appropriate change of variables. It has also been recently tackled by J.A. Iaia, H.A. Warchall \& F.B. Weissler in [18] when the solutions may have an almost constant value on arbitrarily large intervals in $r$ ("loitering on the hilltop"), and then have a node, so that the problem is asymptotically one-dimensional. Here on the contrary, we will take advantage of the lack of regularity, which forces some solutions to have a compact support and therefore localizes the problem in a finite ball. The boundedness of the support of the ground states (non-negative solutions in $H^{1}\left(\mathbb{R}^{d}\right)$ ) has been proved in several papers (see $\left.[5,13,22,16,11]\right)$ and symmetry results based on moving plane methods and comparison techniques (see $[22,11$, $14,15]$ ), are also available even if the nonlinearity is not Lipschitz at $u=0$. However, for sign changing solutions, very little seems to be known in the sublinear case, as quoted for instance in [24] for a related problem (without linear term). Estimates depending on $k$ of $L^{2}$ and $H^{1}$ norms of solutions with $k$ nodes are known in the case of a superlinear and subcritical nonlinearity (see [21]) and one can expect that the nodal structure classifies with the set of the radially symmetric solutions. To end with an open problem, let us mention the question 
of the uniqueness (up to a trivial symmetry $u \mapsto-u$ ) of a solution with a given number of nodes.

Our approach is based on a careful analysis of the following dynamical system in $\mathbb{R}^{2}$, which is equivalent to (2)

$$
\left\{\begin{array}{l}
u^{\prime}=v \\
v^{\prime}=-\frac{d-1}{r} v-f(u) \\
u(0)=\lambda, \quad v(0)=0 .
\end{array}\right.
$$

We evaluate the winding number w.r.t. $(0,0)$ and study for $d>1$ the properties of the flow close to the zero energy curve of the asymptotic autonomous Hamiltonian system (at $r=\infty$ ). Our strategy is to estimate the winding number using the fact that for large $u$, the nonlinearity $f(u)$ is dominated by its linear part, so that the angular velocity in the phase space and the rate of decay of the energy can be estimated. We thus prove that the number of nodes goes to $+\infty$ as $|\lambda|$ goes to $+\infty$. Since solutions of (2) which go to 0 as $r \rightarrow+\infty$ are actually compactly supported, it is possible to build a trapping region at a finite $r$ and thus prove that the number of nodes changes locally w.r.t. the shooting parameter $\lambda=u(0)$ of at most \pm 1 .

The proof of Theorem 1 is given in the next section. It relies (subsection 2.1) on properties which are themselves consequences (subsection 2.3) of some important features of the flow (subsection 2.2). Considerations on existence, regularity, uniqueness (and non uniqueness) of the solutions of (2) and continuity (or lack of continuity) of the flow have been rejected in section 3 .

Notations: To emphasize its dependence on $\lambda$, we will denote by $\left(u_{\lambda}, v_{\lambda}\right)$ the solution of (2). We shall use of the notation $\|(u, v)\|=\sqrt{u^{2}+v^{2}}$ for an arbitrary $(u, v) \in \mathbb{R}^{2}$.

1) A main tool to analyze System (3) is the energy $E_{\lambda}(r)=E\left[u_{\lambda}, v_{\lambda}\right](r)$, where

$$
E[u, v](r)=\frac{1}{2} v^{2}+\frac{1}{2} u^{2}-\frac{|u|^{2(1-\theta)}}{2(1-\theta)}=\frac{1}{2} v^{2}+F(u) .
$$

This energy is decreasing:

$$
\frac{d}{d r} E_{\lambda}(r)=-\frac{d-1}{r} v^{2}(r)<0,
$$

and the only possible limits of $E_{\lambda}$ as $r$ goes to infinity are $F( \pm 1)=-\frac{\theta}{2(1-\theta)}$ or $F(0)=0$, which correspond to the stationary solutions $(u, v)=(0,0)$ or $(u, v)=( \pm 1,0)$ respectively (see Section 3 for more details).

2) If $a \in(0, F(\lambda)]$, we put $\rho_{\lambda}(a)=\inf \left\{r>0: E_{\lambda}(r)=a\right\}$. For simplicity, $\rho_{\lambda}(0)$ is denoted by $\rho_{\lambda}$.

3) We will define $N_{\lambda}\left(R_{1}, R_{2}\right)$ as the number of roots of $u_{\lambda}$ in $\left\{r \in\left(R_{1}, R_{2}\right)\right.$ s.t. $\left.u_{\lambda}^{2}(r)+\left(u_{\lambda}^{\prime}(r)\right)^{2} \neq 0\right\}$ and use the notation $N_{\lambda}(R)=N_{\lambda}(0, R)$. 
4) Our analysis is based on the following sets of initial data:

$$
\begin{aligned}
A_{k} \ni \lambda \Longleftrightarrow & \lambda>0, \lim _{r \rightarrow+\infty} u_{\lambda}(r)= \pm 1, \\
& \left(u_{\lambda}(r), v_{\lambda}(r) \neq(0,0) \forall r \geq 0, \text { and } N_{\lambda}(\infty)=k,\right. \\
I_{k} \ni \lambda \Longleftrightarrow & \lambda>0, \lim _{r \rightarrow+\infty} u_{\lambda}(r)=0 \\
& \text { and } N_{\lambda}\left(\rho_{\lambda}\right)=k \text { for } \rho_{\lambda}=\inf \left\{r>0: u_{\lambda}^{2}(r)+\left(u_{\lambda}^{\prime}(r)\right)^{2}=0\right\} .
\end{aligned}
$$

Note that for any $\lambda \in A_{k}, \lim _{r \rightarrow+\infty} u_{\lambda}(r)=-1$ if $k$ is odd and $\lim _{r \rightarrow+\infty} u_{\lambda}(r)=+1$ if $k$ is even.

\section{Proof of the main result}

We will prove Theorem 1 as follows. First we will deduce it from a list of assertions and get the result by induction. Next, some important features of the flow are established, that are used in the third subsection to prove the assertions.

2.1. Proof of Theorem 1. It relies on the following list of assertions, that will be proved Subsection 2.3. We make the convention: $I_{-1}=\emptyset$.

For all $k \in \mathbb{N}$,

(A1) $A_{k}$ is an open set,

(A2) $A_{k}$ is a bounded set,

(A3) $I_{k}$ is a bounded set,

(A4) $\sup \left(A_{k}\right) \in\left(I_{k-1} \cup I_{k}\right)$,

(A5) $\sup \left(I_{k}\right) \in I_{k}$,

(A6) if $\lambda \in I_{k}$, then there exists $\varepsilon>0$ s.t. $(\lambda-\varepsilon, \lambda-\varepsilon) \subset\left(A_{k} \cup I_{k} \cup A_{k+1}\right)$.

We are going to prove by induction that there exist an increasing sequence $\left(\lambda_{k}\right)_{k \in \mathbb{N}}$ of real positive numbers such that $\lambda_{k} \in I_{k}$. We will specifically prove that for any $k \in \mathbb{I N}$, the three following properties are true:

- for $l=0,1, \ldots k, I_{l} \neq \emptyset$,

- for $l=0,1, \ldots k+1, A_{l} \neq \emptyset$,

- for $l=0,1, \ldots k, \lambda_{l} \leq i_{l}<\lambda_{l+1}$,

where $i_{l}=\sup \left(I_{l}\right)$ and $\lambda_{l}=\sup \left(A_{l}\right)$.

1) The property is true for $k=0 . A_{0}$ is not empty because $1 \in A_{0}$. It is bounded because of (A2). Then $\lambda_{0}=\sup \left(A_{0}\right) \in I_{0}$ because of (A4): $I_{0}$ is not empty, and bounded because of (A3). Let $i_{0}=\sup \left(I_{0}\right)$. Then $i_{0} \in I_{0}$ because of (A5). Let $\varepsilon$ be given by (A6) with $\lambda=i_{0}$. Then $] i_{0}, i_{0}+\varepsilon\left[\subset\left(A_{0} \cup I_{0} \cup A_{1}\right)\right.$. But ]$i_{0}, i_{0}+\varepsilon\left[\cap I_{0}=\emptyset\right.$ by definition of $i_{0}$. And $] i_{0}, i_{0}+\varepsilon\left[\cap A_{0}=\emptyset\right.$ because $i_{0} \geq \lambda_{0}$. This implies $] i_{0}, i_{0}+\varepsilon\left[\subset A_{1}\right.$ and proves that $A_{1}$ is not empty. It is bounded because of (A2). Let $\lambda_{1}=\sup \left(A_{1}\right)$. We have by construction $\lambda_{0} \leq i_{0}<\lambda_{1}$.

2) Proof of the induction. The set $A_{k+1}$ is bounded because of (A2). Then $\lambda_{k+1}=$ $\sup \left(A_{k+1}\right) \in I_{k+1}$ because of (A4), and the assumed inequality $i_{k}<\lambda_{k+1}$. This proves that $I_{k+1} \neq \emptyset$. Let $i_{k+1}=\sup \left(I_{k+1}\right)$. We have of course $\lambda_{k+1} \leq i_{k+1}$. Let $\varepsilon$ be given by (A6) with $\lambda=i_{k+1}$. Then $] i_{k+1}, i_{k+1}+\varepsilon\left[\subset A_{k+1} \cup I_{k+1} \cup A_{k+2}\right.$. 
But $] i_{k+1}, i_{k+1}+\varepsilon\left[\cap I_{k+1}=\emptyset\right.$ by definition of $i_{k+1}$. And $] i_{k+1}, i_{k+1}+\varepsilon\left[\cap A_{k+1}=\emptyset\right.$ because $i_{k+1} \geq \lambda_{k+1}$. This implies $] i_{k+1}, i_{k+1}+\varepsilon\left[\subset A_{k+2}\right.$ and proves that $A_{k+2}$ is not empty. It is bounded because of (A2). Thus we have $\sup \left(A_{k+2}\right)=\lambda_{k+2}>$ $i_{k+1}$ by construction.

\subsection{Main features of the flow.}

Proposition 1. Let $\alpha \in(0,1), r_{\alpha}=\frac{d-1}{2 \alpha \theta}$. Then, for any $a \geq 0$ and $\lambda>0$, we have

$$
N_{\lambda}\left(r_{\alpha}, \rho_{\lambda}(a)\right) \geq \frac{\theta(1-\alpha)}{\pi}\left(\rho_{\lambda}(a)-r_{\alpha}\right) .
$$

Proof. On $] r_{\alpha}, \rho_{\lambda}(a)[$ we have:

$$
\begin{gathered}
\frac{d}{d r}\left(-\operatorname{Arctg}\left(\frac{v}{u}\right)\right)=1+\frac{d-1}{r} \frac{u v}{u^{2}+v^{2}}-\frac{|u|^{2(1-\theta)}}{u^{2}+v^{2}} \\
\geq \theta+\frac{d-1}{r} \frac{u v}{u^{2}+v^{2}}+\frac{2 a(1-\theta)}{u^{2}+v^{2}} \\
\geq \theta-2 \alpha \theta \frac{|u v|}{u^{2}+v^{2}} \geq \theta(1-\alpha) .
\end{gathered}
$$

Integrating this inequality on $] r_{\alpha}, \rho_{\lambda}(a)$ [ gives the result.

Proposition 2. For any $a>0$,

$$
\liminf _{\lambda \rightarrow+\infty} \lambda^{-1 /(d-1)} \rho_{\lambda}(a) \geq \sqrt{d}(8 a)^{-\frac{1}{2(d-1)}} .
$$

Proof. Let $\lambda>2$ and consider a solution of Equation (2). There exists an interval $[0, S(\lambda)]$ such that

$$
\begin{aligned}
& \frac{\lambda}{2} \leq u_{\lambda}(r) \leq \lambda=u_{\lambda}(0) \\
& \quad \text { and } u^{\prime}(r) \leq 0 \quad \forall r \in[0, S(\lambda)], \\
& u_{\lambda}(S(\lambda))=\frac{1}{2} \lambda .
\end{aligned}
$$

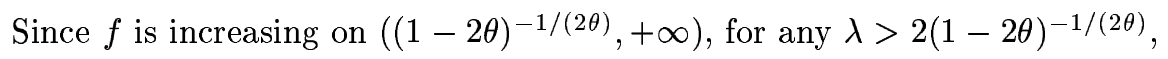

$$
f\left(\frac{\lambda}{2}\right) \leq f(u(r)) \leq f(\lambda) \quad \forall r \in[0, S(\lambda)]
$$

and from the identity

$$
\frac{\lambda}{2}=u_{\lambda}(0)-u_{\lambda}(S(\lambda))=\int_{0}^{S(\lambda)}\left(\int_{0}^{r} f(u(s)) s^{d-1} d s\right) \frac{d r}{r^{d-1}},
$$

we get

$$
\frac{1}{2 d}(S(\lambda))^{2} f\left(\frac{\lambda}{2}\right) \leq \frac{\lambda}{2} \leq \frac{1}{2 d}(S(\lambda))^{2} f(\lambda) .
$$

This gives, using the fact that $f(u) \sim u$ as $u \rightarrow+\infty$, the estimate

$$
\sqrt{d} \leq \liminf _{\lambda \rightarrow+\infty} S(\lambda) \leq \limsup _{\lambda \rightarrow+\infty} S(\lambda) \leq \sqrt{2 d} .
$$


Moreover, by definition of $E_{\lambda}$,

$$
\frac{d}{d r}\left(E_{\lambda}(r)-m\right)=-2 \frac{d-1}{r}\left(E_{\lambda}(r)-F\left(u_{\lambda}(r)\right)\right) \geq-2 \frac{d-1}{r}\left(E_{\lambda}(r)-m\right)
$$

with $m=F( \pm 1)=\min _{u \in \mathbb{R}} F(u)=-\frac{\theta}{2(1-\theta)}$. Integrating between $S(\lambda)$ and $r>S(\lambda)$ gives

$$
r^{2(d-1)}\left(E_{\lambda}(r)-m\right) \geq(S(\lambda))^{2(d-1)}\left(E_{\lambda}(S(\lambda))-m\right) \quad \forall r>S(\lambda),
$$

which combined with

$$
E_{\lambda}(S(\lambda)) \geq F\left(\frac{\lambda}{2}\right)=\frac{\lambda^{2}}{8}-\frac{\lambda^{2(1-\theta)}}{2^{3-2 \theta}(1-\theta)} \sim \frac{\lambda^{2}}{8} \quad \text { as } \lambda \rightarrow+\infty
$$

and (6) provides the estimate

$$
\begin{gathered}
a=E_{\lambda}\left(\rho_{\lambda}(a)\right)=m \\
+\left(\frac{\sqrt{d}}{r}\right)^{2(d-1)}\left(\frac{\lambda^{2}}{8}-\frac{\lambda^{2(1-\theta)}}{2^{3-2 \theta}(1-\theta)}-m\right) \\
\geq m+\left(\frac{\sqrt{d}}{r}\right)^{2(d-1)} \frac{\lambda^{2}}{8}(1+o(1))
\end{gathered}
$$

as $\lambda \rightarrow+\infty$. Estimate (5) immediately follows.

Corollary 1. Let $N_{\lambda}(\infty)$ be the number of nodes of a solution of (2) with initial data $u(0)=\lambda$. Then

$$
\lim _{\lambda \rightarrow+\infty} N_{\lambda}(\infty)=+\infty
$$

Proof. Let $\alpha \in(0,1), a>0$ be given. By Proposition 1,

$$
N_{\lambda}(\infty) \geq N_{\lambda}\left(r_{\alpha}, \rho_{\lambda}(a)\right) \geq \frac{\theta(1-\alpha)}{\pi}\left(\rho_{\lambda}(a)-r_{\alpha}\right) .
$$

For $\lambda$ large, $\rho_{\lambda}(a) \geq \frac{1}{2} \sqrt{d}(8 a)^{-\frac{1}{2(d-1)}} \lambda^{1 /(d-1)}$ (by Proposition 2), which proves that

$$
\liminf _{\lambda \rightarrow+\infty} \frac{N_{\lambda}(\infty)}{\lambda^{\frac{1}{d-1}}} \geq \sqrt{d}(8 a)^{-\frac{1}{2(d-1)}} .
$$

Proposition 3. For any $k \in N$, there exists an $\varepsilon>0$ such that, if $N_{\lambda}(R)=k$ and $u_{\lambda}^{2}(R)+v_{\lambda}^{2}(R)<\varepsilon^{2}$, then $\lambda$ belongs to $A_{k} \cup I_{k} \cup A_{k+1}$.

Proof. The decay of the energy $E_{\lambda}(r)$ makes it obvious if $\left(u_{\lambda}(R), v_{\lambda}(R)\right) \in\{E \leq$ $0\}$. By symmetry, it is enough to prove the existence of an $\varepsilon>0$, small enough, for

$$
\left(u_{\lambda}(R), v_{\lambda}(R)\right) \in\{\|(u, v)\|<\varepsilon\} \cap\{E>0\} \cap\left\{0<v<\frac{1}{2} \sqrt{\frac{\theta}{1-\theta}}\right\} .
$$


For this purpose, we consider the trapping region $\Omega=\left\{0<v<\sqrt{\frac{\theta}{1-\theta}}\right\} \cap\{E>0\}$ (see Fig. 3, Section 4). Let $\alpha \in(0,1)$ (see Proposition 1 ). We claim that if $\varepsilon$ (which therefore depends on $k, \alpha$ and $\theta$ ) is defined by the condition:

$$
\|(u, v)\|<\varepsilon \Longrightarrow E(u, v)<\frac{(d-1) \theta}{4(1-\theta)} \frac{1}{\frac{\pi(k+1)}{\theta(1-\alpha)}+r_{\alpha}} \frac{1}{4} \sqrt{\frac{\theta}{1-\theta}},
$$

then $\left(u_{\lambda}, v_{\lambda}\right)$ exits the region $\Omega$ crossing the boundary at $\{E=0\}$. Assuming the claim we have:

- If $u_{\lambda}(R)<0$, and the boundary is crossed at $u<0$, then $\lambda$ belongs to $A_{k}$.

- If $u_{\lambda}(R)<0$, and the boundary is crossed at $u=0$, then $\lambda$ belongs to $I_{k}$.

- If $u_{\lambda}(R)<0$, and the boundary is crossed at $u>0$, then $\lambda$ belongs to $A_{k+1}$.

- If $u_{\lambda}(R) \geq 0$, the boundary can only be crossed at $u>0$ because $u^{\prime}=v>0$.

In that case $\lambda$, belongs to $A_{k}$.

We are now going to prove the claim, by contradiction. Assume $\left(u_{\lambda}, v_{\lambda}\right)$ exits $\Omega$ crossing the boundary at $\left\{v=\sqrt{\frac{\theta}{1-\theta}}\right\}$. Let $R^{\prime \prime}$ be the value of $r$ at the crossing. Then $E_{\lambda}\left(R^{\prime \prime}\right)>0$. Moreover, if $0<\alpha<1$, we have by Proposition 1 :

$k=N_{\lambda}(R) \leq N_{\lambda}\left(R^{\prime \prime}\right) \leq k+1 \quad$ and $\quad N_{\lambda}\left(R^{\prime \prime}\right) \geq N_{\lambda}\left(r_{\alpha}, R^{\prime \prime}\right) \geq \frac{\theta(1-\alpha)}{\pi}\left(R^{\prime \prime}-r_{\alpha}\right)$.

This implies

$$
R^{\prime \prime} \leq \frac{\pi(k+1)}{\theta(1-\alpha)}+r_{\alpha} .
$$

Let $R^{\prime}=\sup \left\{r \in\left(R, R^{\prime \prime}\right): v_{\lambda}(r)<\frac{1}{2} \sqrt{\frac{\theta}{1-\theta}}\right\}$, which is well defined because of Condition (8). Inequality (10) gives:

$$
E_{\lambda}\left(R^{\prime}\right)-E_{\lambda}\left(R^{\prime \prime}\right)=\int_{R^{\prime}}^{R^{\prime \prime}} \frac{d-1}{r} v_{\lambda}^{2}(r) d r \geq \frac{(d-1) \theta}{4(1-\theta)} \frac{1}{\frac{\pi(k+1)}{\theta(1-\alpha)}+r_{\alpha}}\left(R^{\prime \prime}-R^{\prime}\right) .
$$

Thus we have

$$
E_{\lambda}\left(R^{\prime \prime}\right) \leq E_{\lambda}\left(R^{\prime}\right)-\frac{(d-1) \theta}{4(1-\theta)} \frac{1}{\frac{\pi(k+1)}{\theta(1-\alpha)}+r_{\alpha}}\left(R^{\prime \prime}-R^{\prime}\right) .
$$

On the other hand, on $] R^{\prime}, R^{\prime \prime}\left[,\left|u_{\lambda}\right| \leq 1\right.$, and $v_{\lambda}^{\prime}=-\frac{d-1}{r} v_{\lambda}-u_{\lambda}+\left|u_{\lambda}\right|^{-2 \theta} u_{\lambda} \leq$ $\left|u_{\lambda}\right|+\left|u_{\lambda}\right|^{1-2 \theta} \leq 2$. This gives the estimate

$$
\frac{1}{2} \sqrt{\frac{\theta}{1-\theta}}=v_{\lambda}\left(R^{\prime \prime}\right)-v_{\lambda}\left(R^{\prime}\right) \leq 2\left(R^{\prime \prime}-R^{\prime}\right) .
$$
get

Combining inequalities (11) and (12) and using the decay of the energy, we

$$
\begin{aligned}
0<E_{\lambda}\left(R^{\prime \prime}\right) & \leq E_{\lambda}\left(R^{\prime}\right)-\frac{(d-1) \theta}{4(1-\theta)} \frac{1}{\frac{\pi(k+1)}{\theta(1-\alpha)}+r_{\alpha}} \frac{1}{4} \sqrt{\frac{\theta}{1-\theta}} \\
& \leq E_{\lambda}(R)-\frac{(d-1) \theta}{4(1-\theta)} \frac{1}{\frac{\pi(k+1)}{\theta(1-\alpha)}+r_{\alpha}} \frac{1}{4} \sqrt{\frac{\theta}{1-\theta}} .
\end{aligned}
$$

This gives a lower bound on $E_{\lambda}(R)$ which contradicts the definition (9) of $\varepsilon$ and therefore proves the claim. 


\subsection{Proof of Assertions (A1)-(A6).}

Assertion (A1) For any $k \in \mathbb{N}$, the set $A_{k}$ is an open set.

Proof. Let $\lambda_{0} \in A_{k}$. By Proposition 6, $\rho_{\lambda_{0}}=\inf \left\{r>0: E_{\lambda_{0}}(r)=0\right\}<\infty$. Because of the energy decay (4), $E_{\lambda_{0}}\left(\rho_{\lambda_{0}}+1\right)<0$ and the regularity of the flow (see Corollary 2) in a neighbourhood of $\left(u_{\lambda_{0}}, v_{\lambda_{0}}\right)$ then gives the existence of an $\varepsilon_{1}>0$ such that

$$
\left|\lambda-\lambda_{0}\right|<\varepsilon_{1} \Longrightarrow E_{\lambda}\left(\rho_{\lambda_{0}}+1\right)<0 \text {. }
$$

Because of Corollary 2 again, $u_{\lambda}$ does not hit $(0,0)$ for $\varepsilon_{1}$ small enough. This proves that corresponding the $\lambda$ belong to $\cup_{l \in \mathbb{N}} A_{l}$.

Moreover, the set of $r$ in $] 0, \rho_{\lambda_{0}}+1\left[\right.$, such that $u_{\lambda_{0}}(r)=0$, is finite by Proposition 6 , and for such an $r$, we have $u_{\lambda_{0}}^{\prime}(r)=v_{\lambda_{0}}(r) \neq 0$. This proves that $\{u=0\}$ is crossed transversally by $\left(u_{\lambda_{0}}, v_{\lambda_{0}}\right)$. Corollary 2 gives the existence of an $\varepsilon_{2}>0$ such that if $\left|\lambda-\lambda_{0}\right|<\varepsilon_{2}$, then $u_{\lambda}$ has the same number of nodes as $u_{\lambda_{0}}$ on $] 0, \rho_{\lambda_{0}}+1\left[\right.$. The decay of the energy then implies that $u_{\lambda}$ has no root on $\left[\rho_{\lambda_{0}}+1, \infty[\right.$.

If $\left|\lambda-\lambda_{0}\right|<\min \left(\varepsilon_{1}, \varepsilon_{2}\right)$, then $\lambda \in A_{k}$ and the assertion is proved.

Assertion (A2) For any $k \in \mathbb{N}$, the set $A_{k}$ is bounded.

Proof. If Assertion (A2) was false, there would be an unbounded increasing sequence $\left(\lambda^{j}\right)_{j \in \mathbb{N}}$ of elements of $A_{k}$ for which, by definition of $A_{k}, N_{\lambda^{j}}(\infty)=k$, a contradiction with Corollary 1.

Assertion (A3) For any $k \in \mathbb{N}$, the set $I_{k}$ is bounded.

Proof. It is the same as for (A2).

Assertion (A4) For any $k \in \mathbb{N}$, one has: $\sup \left(A_{k}\right) \in\left(I_{k-1} \cup I_{k}\right)$.

Proof. Let $\lambda_{k}=\sup \left(A_{k}\right)$. Assertion (A1) tells that the sets $A_{n}$ are open disjoint sets. This implies $\lambda_{k} \notin \cup A_{n}$. Because of Proposition 6, $\mathbb{R}_{+}=\{0\} \cup\left(\cup I_{l}\right) \cup\left(\cup A_{n}\right)$, so $\lambda_{k} \in \cup I_{n}$. Let $m$ be such that $\lambda_{k} \in I_{m}$ and consider $\beta>0$ such that $u_{\lambda_{k}}\left(\rho_{\lambda_{k}}-\right.$ $\beta) \neq 0$ and $u_{\lambda_{k}}$ has $m$ roots on $] 0, \rho_{\lambda_{k}}-\beta$. Moreover the continuity of $\left(u_{\lambda_{k}}, v_{\lambda_{k}}\right)$ enables us to choose $\beta>0$ such that $u_{\lambda_{k}}^{2}\left(\rho_{\lambda_{k}}-\beta\right)+v_{\lambda_{k}}^{2}\left(\rho_{\lambda_{k}}-\beta\right)<\frac{1}{2} \varepsilon^{2}$, where $\varepsilon$ is given by Proposition 3, and $0<\left|v_{\lambda_{k}}\right|<\frac{1}{2} \sqrt{\frac{\theta}{1-\theta}}$. Because of the regularity of the flow in a neighbourhood of $\left(u_{\lambda_{k}}(r), v_{\lambda_{k}}(r)\right)_{r \in\left[0, \rho_{\lambda_{k}}-\beta\right]}$ (see Corollary 2), there exists an $\eta>0$ such that for $\left|\lambda_{k}-\lambda\right|<\eta, u_{\lambda}^{2}\left(\rho_{\lambda}-\beta\right)+v_{\lambda}^{2}\left(\rho_{\lambda}-\beta\right)<\frac{1}{2} \varepsilon^{2}$, $0<\left|v_{\lambda}\right|<\frac{1}{2} \sqrt{\frac{\theta}{1-\theta}}$ and $u_{\lambda}$ has $m$ roots on $] 0, \rho_{\lambda}-\beta[$ (this is because $u=0$ is always crossed transversally if crossed with $v \neq 0$ : see the proof of Assertion (A1) for more details). Definition of $\lambda_{k}$ gives such a $\lambda$ in $A_{k}$. Proposition 3 tells that $\lambda \in\left(A_{m} \cup I_{m} \cup A_{m+1}\right)$. This implies $k=m$ or $k=m+1$, and the assertion is proved.

Assertion (A5) For any $k \in \mathbb{N}$, one has: $\sup \left(I_{k}\right) \in I_{k}$.

Proof. The same proof as for Assertion (A4) applies mutatis mutandis.

Assertion (A6) Let $k \in \mathbb{N}$ and $\lambda \in I_{k}$. There exists an $\varepsilon>0$ such that

$$
(\lambda-\varepsilon, \lambda+\varepsilon) \subset\left(A_{k} \cup I_{k} \cup A_{k+1}\right) .
$$


Proof. As in the proof of Assertion (A4), one can choose a $\beta>0$ such that $u_{\lambda}\left(\rho_{\lambda}-\beta\right) \neq 0, u_{\lambda}$ has $k$ roots on $] 0, \rho_{\lambda}-\beta\left[\right.$, and $u_{\lambda}^{2}\left(\rho_{\lambda}-\beta\right)+v_{\lambda}^{2}\left(\rho_{\lambda}-\beta\right)<\frac{\varepsilon^{2}}{2}$, where $\varepsilon$ is given by Proposition 3. Regularity of the flow in a neighbourhood of $\left(u_{\lambda}(r), v_{\lambda}(r)\right)_{r \in\left[0, \rho_{\lambda}-\beta\right]}$ gives an eventually smaller $\varepsilon>0$ such that $|\mu-\lambda|<\varepsilon$ implies $u_{\mu}^{2}\left(\rho_{\lambda}-\beta\right)+v_{\mu}^{2}\left(\rho_{\lambda}-\beta\right)<\frac{1}{2} \varepsilon^{2}, 0<\left|v_{\mu}\right|<\frac{1}{2} \sqrt{\frac{\theta}{1-\theta}}$ and $u_{\mu}$ has $k$ roots on $] 0, \rho_{\lambda}-\beta[$ (same argument as for Assertion (A4)). By Proposition 3, $\mu \in\left(A_{k} \cup I_{k} \cup A_{k+1}\right)$, and the assertion is proved.

\section{Additional results}

3.1. Existence, uniqueness, continuity of the flow. In this subsection, we collect results which are standard for regular nonlinearities. The proofs in our case are similar. We give them for the sake of completeness.

Proposition 4. [Existence]. For any $\lambda$ in $\mathbb{R}$, (2) has a solution of class $C^{2}$ on $\mathbb{R}^{+}$.

Proof. For $\lambda \in \mathbb{R}$, we consider the regularized problem

$$
\left\{\begin{array}{l}
u^{\prime \prime}+\frac{d-1}{r} u^{\prime}+F_{\epsilon}^{\prime}(u)=0 \\
u(0)=\lambda, \quad v(0)=0
\end{array}\right.
$$

Here $F_{\epsilon}(u)=\frac{u^{2}}{2}-\frac{\left(\epsilon^{2}+u^{2}\right)^{1-\theta}}{1-\theta}$ for $\epsilon>0$. The function $F_{\epsilon}^{\prime}(u)=u-\frac{u}{\left(\epsilon^{2}+u^{2}\right)^{\theta}}$ is smooth, so by standard methods, any local solution has $C^{\infty}$ regularity. Moreover

$$
\frac{d}{d r}\left[\frac{1}{2} u^{\prime 2}(r)+F_{\epsilon}(u(r))\right]=-\frac{d-1}{r} u^{\prime 2}(r)<0 .
$$

So no blow-up can occur and any solution of (13) is therefore uniformly bounded in $C^{1}$. This proves the global existence of a unique solution of (13) in $C^{1}$. Moreover

$$
\frac{1}{2} u^{\prime 2}(r)+F_{\epsilon}(u(r)) \leq F_{\epsilon}(\lambda) \leq F(\lambda) .
$$

So any solution of (13) is uniformly (with respect to $\epsilon$ ) bounded in $W^{1, \infty}\left(\mathbb{R}^{+}\right)$, and also in $W^{2, \infty}\left(\mathbb{R}^{+}\right)$because of (13), which allows to go to the limit and get a global existence result for (2).

Remark 1. A main open problem is the uniqueness of the solution of (2) with limit 0 as $r \rightarrow+\infty$ and a given arbitrary number of nodes (up to the trivial change of sign corresponding to $-u_{-\lambda}=u_{\lambda}$ ).

In the remaining, only $\lambda>0$ will be considered.

Proposition 5. [Regularity]. Any radial solution of (1) on $\mathbb{R}^{d}$ in the distribution sense has $C^{2}$ regularity. 
Proof. Standard results show that any solution of (2) in the distribution sense is locally $C^{\infty}$ whenever $u \neq 0$. It is continuous in a neighborhood of any root, except maybe at $r=0$. Next, using the fact that $r \mapsto r^{d-1} u^{\prime}(r)$ is a primitive function of $r \mapsto-r^{d-1} f(u(r))$, one proves that bounded solutions are of class $C^{2}$ on $(0,+\infty)$. For radial solutions of $(1)$, a singularity could only occur at $r=0$. But in this case, the singularity is removed by the choice of the Cauchy data as follows.

The behaviour of the solution near $r=0$ can indeed be investigated by standard techniques (see for instance [4] and references therein). Let $w$ be defined by $u(r)=r^{1 / \theta} w(t)$ with $t=-\log r$. The problem is reduced to the study of the asymptotic behaviour of the solution of

$$
w^{\prime \prime}+\left(2-d-\frac{2}{\theta}\right) w^{\prime}-|w|^{-2 \theta} w+\frac{1+\theta(d-2)}{\theta^{2}} w+e^{-2 t} w=0,
$$

which behaves like $C e^{\nu t}$ as $t \rightarrow+\infty$, for some $C \neq 0$, and $\nu$ given by

$$
\nu^{2}+\left(2-d-\frac{2}{\theta}\right) \nu+\frac{1+\theta(d-2)}{\theta^{2}}=0 .
$$

Then either $\nu=\frac{1}{\theta}$ and the solution is bounded, or $\nu=d-2+\frac{1}{\theta}$ and the singularity at the origin is not removable. Radial solutions of (1) in the distributions sense are therefore bounded.

Proposition 6. [Asymptotics] (Fig. $1 \& 2$, Section 4). Any solution $u_{\lambda}$ of (2) has a finite number of nodes $N_{\lambda}(\infty)$. If $N_{\lambda}(\infty) \geq 1$, then $N_{\lambda}(\infty)=N_{\lambda}\left(\rho_{\lambda}\right)$, where $\rho_{\lambda}=\inf \left\{r>0: E_{\lambda}(r)=0\right\}$ is finite. As $r \rightarrow+\infty$ the solution $u_{\lambda}$ converges to a limit which is

- either \pm 1 , and in this case $u_{\lambda}$ does not change sign on $\left(\rho_{\lambda}^{\prime}, \infty\right)$, where $\rho_{\lambda}^{\prime}=$ $\sup \left\{r>0: E_{\lambda}(r)=0\right\}<\infty$,

- or 0 , and in this case the solution is compactly supported and belongs to $H^{1}\left(\mathbb{R}^{d}\right)$.

Proof. Let us prove that the only possible limits of $(u, v)$ are either $( \pm 1,0)$ or $(0,0)$, which correspond to stationary points of System (3) in the phase space. The sequence $\left(u_{\lambda}(\cdot+n)\right)_{n \in \mathbb{N}}$ indeed converges (up to the extraction of a subsequence indexed by $\left.\left(n_{k}\right)_{k \in \mathbb{N}}\right)$ weakly in $W^{2, \infty}\left(\mathbb{R}^{+}\right)$to some limiting function $\bar{u}$ which is a solution of

$$
\bar{u}^{\prime \prime}+f(\bar{u})=0
$$

such that $\frac{1}{2} \bar{u}^{\prime 2}+F(\bar{u})=\lim _{r \rightarrow+\infty} E_{\lambda}(r)$ (see for instance [10] for a general exposition of this type of results). Asymptotically as $k \rightarrow+\infty$,

$$
\int_{1}^{+\infty} \frac{d-1}{r} u_{\lambda}^{\prime 2}\left(r+n_{k}\right) d r \sim \int_{1}^{+\infty} \frac{d-1}{r} \bar{u}^{2}(r) d r,
$$

so that $u_{\lambda}^{\prime}\left(\cdot+n_{k}\right) \rightarrow 0 \equiv \bar{u}^{\prime}$. It is then easy to check that the only constant solutions of Equation (14) are 0 and \pm 1 .

It also readily follows from energy estimates that if for some $\lambda \in \mathbb{R}$, there exists an $r_{0}>0$ such that $E_{\lambda}\left(r_{0}\right)=0$ with $\left(u_{\lambda}\left(r_{0}\right), u_{\lambda}^{\prime}\left(r_{0}\right)\right) \neq(0,0)$, where $u_{\lambda}$ is a solution of Equation (2), then the only possible limit of $u_{\lambda}$ as $r \rightarrow+\infty$ is \pm 1 and $N_{\lambda}(\infty)=N_{\lambda}\left(r_{0}\right)$. 
Moreover, the fact that any solution $u_{\lambda}$ of (2) which has limit 0 is actually compactly supported has been proved by C. Cortazár, M. Elgueta \& P. Felmer in [11] for ground states (also see [27]). However their proof does not use positivity. For the completeness of the paper, we give here the argument (in the special case of radial solutions).

Let $\bar{u}$ be implicitely defined by

$$
\sqrt{2} r=\int_{\bar{u}(r)}^{a_{\theta}} \frac{d s}{\sqrt{-F(s)}} \quad \text { with } \quad a_{\theta}=(1-2 \theta)^{-\frac{1}{2 \theta}},
$$

where $F(u)=\frac{1}{2} u^{2}-\frac{|u|^{2(1-\theta)}}{2(1-\theta)}$. The function $s \mapsto(-F(s))^{-1 / 2}$ is integrable at $s=0_{+}$since $\theta \in\left(0, \frac{1}{2}\right)$. The function $\bar{u}$ solves

$$
\left\{\begin{array}{l}
\bar{u}^{\prime \prime}+f(\bar{u})=0 \quad \text { on } \quad\left(0, A_{\theta}\right) \\
\bar{u}(0)=a_{\theta} \text { and } \quad \bar{u}\left(A_{\theta}\right)=\bar{u}^{\prime}\left(A_{\theta}\right)=0
\end{array}\right.
$$

where $A_{\theta}=\frac{1}{\sqrt{2}} \int_{0}^{a_{\theta}} \frac{d s}{\sqrt{-F(s)}}$. We may extend $\bar{u}$ by 0 on $\left(A_{\theta},+\infty\right)$. Consider a solution $u_{\lambda}$ of (2) such that $\lim _{r \rightarrow+\infty} u_{\lambda}(r)=0$ and let $R_{\lambda}$ be such that on $\left|u_{\lambda}\right| \leq a_{\theta}$ on $\left(R_{\lambda},+\infty\right)$. We have

$$
u_{\lambda}\left(R_{\lambda}\right) \leq a_{\theta}=\bar{u}\left(r-R_{\lambda}\right)_{\mid r=R_{\lambda}}
$$

and

$$
\lim _{r \rightarrow+\infty}\left|u_{\lambda}(r)-\bar{u}\left(r-R_{\lambda}\right)\right|=0 .
$$

If the maximum of $u_{\lambda}-\bar{u}\left(\cdot-R_{\lambda}\right)$ is positive, then it is reached at some point $r_{\lambda} \in\left(R_{\lambda},+\infty\right)$, for which we may write

$$
\begin{aligned}
0 \leq-\Delta & \left(u_{\lambda}\left(r_{\lambda}\right)-\bar{u}\left(r_{\lambda}-R_{\lambda}\right)\right) \\
= & \frac{d-1}{r_{\lambda}} \bar{u}^{\prime}\left(r_{\lambda}\right)+f\left(u_{\lambda}\left(r_{\lambda}\right)\right)-f\left(u_{\lambda}\left(r_{\lambda}\right)-\left(u_{\lambda}\left(r_{\lambda}\right)-\bar{u}\left(r_{\lambda}\right)\right)\right)<0 .
\end{aligned}
$$

This is because $\bar{u}$ is nonincreasing and $f$ is strictly decreasing on $\left(-a_{\theta}, a_{\theta}\right)$, and leads to a contradiction. Thus

$$
u_{\lambda}-\bar{u}\left(\cdot-R_{\lambda}\right) \leq 0 \quad \text { on } \quad\left(R_{\lambda},+\infty\right) .
$$

This result is also true for $-u_{\lambda}=u_{-\lambda}$ :

$$
u_{\lambda}+\bar{u}\left(\cdot-R_{\lambda}\right) \geq 0 \quad \text { on } \quad\left(R_{\lambda},+\infty\right) .
$$

It follows that $u_{\lambda}(r) \equiv 0$ for any $r>R_{\lambda}+A_{\theta}$.

Proposition 7. [Local uniqueness]. The flow $r \mapsto(u(r), v(r))$ defined by

$$
\left\{\begin{array}{l}
u^{\prime}=v \\
v^{\prime}=-\frac{d-1}{r} v-f(u)
\end{array}\right.
$$

is uniquely defined on $\mathbb{R}^{+}$as long as it takes its values in $\mathbb{R}^{2} \backslash\{(0,0)\}$. 
Proof. It is enough to prove local uniqueness. We know that the flow is unique as long as $f$ is locally Lipschitz, i.e. $u(r) \neq 0$. Assume that for some $\epsilon>0, r_{0}>0$, there are two solutions which coincide on $\left(r_{0}-\epsilon, r_{0}\right)$, differ on $\left(r_{0}, r_{0}+\epsilon\right)$ and are such that $u_{1}\left(r_{0}\right)=u_{2}\left(r_{0}\right)=u_{0}$ and $u_{1}^{\prime}\left(r_{0}\right)=u_{2}^{\prime}\left(r_{0}\right)=v_{0}$. Then $u_{0}=0$. It is enough to prove that $v_{0}=0$. If this was not the case, using a local inversion theorem, we could consider two functions $r_{1}$ and $r_{2}$ such that for $|t|$ small, $r_{1}^{\prime}$ and $r_{2}^{\prime}$ have no root and

$$
u_{i}\left(r_{i}(t)\right)=t \quad(i=1,2)
$$

(Proposition 5 allows it). The functions $r_{1}$ and $r_{2}$ would solve

$$
\left\{\begin{array}{l}
-\frac{r^{\prime \prime}}{r^{\prime 3}}+\frac{d-1}{r r^{\prime}}+f(t)=0 \\
r(0)=r_{0} \text { and } r^{\prime}(0)=v_{0}^{-1}
\end{array}\right.
$$

The difference $\Delta(t)=r_{2}(t)-r_{1}(t)$ would then solve a second order equation with smooth coefficients, with $\Delta(t) \equiv 0$ on a half neighbourhood of 0 . By unique continuation, $\Delta(t) \equiv 0$ everywhere, which contradicts the assumption $v_{0} \neq 0$.

As a straightforward consequence, we obtain the continuity of the flow with respect to the initial data in $\mathbb{R}^{2} \backslash\{(0,0)\}$.

Corollary 2. [Continuity of the flow]. Let $(\bar{u}, \bar{v})$ solve $(15)$ on $\left(r_{0},+\infty\right)$ for some $r_{0} \geq 0$ (if $r_{0}=0$, we further assume $\bar{v}(0)=0$ ). Let $r>r_{0}$ be such that for any $s \in\left[r_{0}, r\right],|\bar{u}(s)|^{2}+|\bar{v}(s)|^{2} \neq 0$. Then for any given $\epsilon>0$, there exists $\delta>0$ such that, if $(u, v)$ solves (15) with $u\left(r_{0}\right)=u_{0}$ and $v\left(r_{0}\right)=v_{0}$, and $\left|u_{0}-\bar{u}\left(r_{0}\right)\right|^{2}+\left|v_{0}-\bar{v}\left(r_{0}\right)\right|^{2}<\delta$, then $|u(s)-\bar{u}(s)|^{2}+|v(s)-\bar{v}(s)|^{2}<\epsilon$ for any $s \in\left[r_{0}, r\right]$ (if $r_{0}=0$, we further assume $v_{0}=0$ ).

Proof. This is done by contradiction. Let $\left(u_{n}, v_{n}\right)_{n \in \mathbb{N}}$ be a sequence of solutions of (15) such that $\lim _{n \rightarrow \infty}\left(\left|u_{n}-\bar{u}\right|^{2}+\left|v-\bar{v}_{n}\right|^{2}\right)_{\mid r=r_{0}}=0$ and, for some $s \in\left[r, r_{0}\right]$, $\lim _{n \rightarrow \infty}\left(\left|u_{n}-\bar{u}\right|^{2}+\left|v_{n}-\bar{v}\right|^{2}\right)_{\mid r=\bar{s}}=\epsilon_{0}>0$. Exactly as in proof of Proposition 4, the uniform bound in $W^{2, \infty}\left(r_{0}, r\right)$ implies that the limit of $\left(u_{n}, v_{n}\right)$ is a solution with initial data $\left(\bar{u}\left(r_{0}\right), \bar{v}\left(r_{0}\right)\right)$ at $r_{0}$. The solution differs from $(\bar{u}, \bar{v})$, which contradicts uniqueness (Proposition 7).

Uniqueness and continuity with respect to the initial data are lost for a solution which hits $(0,0)$.

Proposition 8. Let $r_{0} \geq 0$. There exists a solution $u$ on $\left(r_{0},+\infty\right)$ with $u(r)>0$ for $r>r_{0}$, which solves

$$
\left\{\begin{array}{l}
u^{\prime \prime}+\frac{d-1}{r} u^{\prime}+f(u)=0 \\
u\left(r_{0}\right) \stackrel{=}{=} u^{\prime}\left(r_{0}\right)=0
\end{array}\right.
$$

$\underline{\text { Proof. }}$ To prove this, we may consider solutions on $\left(r_{0},+\infty\right)$ of the regularized equation

$$
\left\{\begin{array}{l}
u_{\epsilon}^{\prime \prime}+\frac{d-1}{r} u_{\epsilon}^{\prime}+F_{\epsilon}^{\prime}\left(u_{\epsilon}\right)=0 \\
u_{\epsilon}\left(r_{0}\right) \stackrel{=}{=}, \quad u_{\epsilon}^{\prime}\left(r_{0}\right)=\epsilon
\end{array}\right.
$$


We take the limit $\epsilon \rightarrow 0$ as in Proposition 4. It is enough to prove that the limit is not identically 0 . Using the decay of the energy, it is clear that

$$
\left|u_{\epsilon}^{\prime}\right|<\sqrt{2\left(\epsilon^{2}-F_{\epsilon}\left(u_{\epsilon}\right)\right)} .
$$

For any $\delta>0$ such that $u_{\epsilon}^{\prime}>0$ on $\left(r_{0}, r_{0}+\delta\right)$ uniformly in $\epsilon$, we have therefore

$$
r_{0}+\delta>r_{0}+\int_{0}^{u_{\epsilon}(\delta)} \frac{d w}{\sqrt{2\left(\epsilon^{2}-F_{\epsilon}(w)\right)}} .
$$

This forbids the convergence of $u_{\epsilon}$ to 0 on $\left(r_{0}, r_{0}+\delta\right)$ and moreover gives the estimate

$$
\delta \geq \int_{0}^{u(\delta)} \frac{d w}{\sqrt{-2 F(w)}} .
$$

The energy decay implies that the sign of $u$ is constant.

Remark 2. [Non-uniqueness] A similar existence result also holds for negative solutions on $\left(r_{0},+\infty\right)$. The fact that we can extend a non-negative (or nonpositive) solution of Equation (16) on $\left(0, r_{0}\right)$ by 0 or by a compactly supported solution (with support in $\left(0, r_{0}\right)$, which is possible for $r_{0}>0$ big enough) clearly contradicts the uniqueness and the continuity with respect to the initial data. One can even extend a non-negative compactly supported solution $u_{\bar{\lambda}}$ by a positive solution of Equation (16). Such a solution is clearly neither the limit as $\lambda \rightarrow \bar{\lambda}$ of solutions $u_{\lambda}$ of (2) nor a solution that can be approximated by a simple regularization procedure like the one used in the proof of Proposition 4 .

However, one can prove that all radial solutions are either the ones constructed in Proposition 4 or combinations of compactly supported solutions and solutions of (16).

\subsection{Further remarks.}

a) Nonlinear eigenvalues.

Consider radial solutions in $\mathbb{R}^{d}$ of

$$
\Delta v+\mu v-|v|^{-2 \theta} v=0
$$

For $\mu>0$, the scaling given by $v(x)=\mu^{-\frac{1}{2 \theta}} u(\sqrt{\mu} x)$ reduces the problem to Equation (1). This means that to each radial solution of (1), we may associate a branch of solutions of (17) parametrized by $\mu$, namely $\mu \mapsto\left(\mu, \mu^{-\frac{1}{2 \theta}}\|u\|_{L^{\infty}\left(\mathbb{R}^{d}\right)}\right)$ in $(0,+\infty) \times L^{\infty}\left(\mathbb{R}^{d}\right)$, and $\mu \mapsto\left(\mu, \mu^{-\left(\frac{d}{4}+\frac{1}{2 \theta}\right)}\left(\mu\|\nabla u\|_{L^{2}\left(\mathbb{R}^{d}\right)}^{2}+\|u\|_{L^{2}\left(\mathbb{R}^{d}\right)}^{2}\right)^{1 / 2}\right)$ in $(0,+\infty) \times H^{1}\left(\mathbb{R}^{d}\right)$ provided $u$ is compactly supported. Note that, as mentioned in the introduction, the case $\mu=\omega^{2}$ is the standing wave problem in the wave equation. For $\mu \leq 0$, no bounded solution in $L^{\infty}\left(\mathbb{R}^{d}\right)$ or in $H^{1}\left(\mathbb{R}^{d}\right)$ may exist, except $v \equiv 0$ (see Fig. 4, Section 4).

The proof of non-existence of solutions in $H^{1}\left(\mathbb{R}^{d}\right)$ is easy. Let $v \in H^{1}\left(\mathbb{R}^{d}\right)$ be a solution of $\Delta v=v+|v|^{-2 \theta} v$. Multiplying the equation by $v$, we get

$$
-\int_{\mathbb{R}^{d}}|\nabla v|^{2} d x=\int_{\mathbb{R}^{d}}\left(|v|^{2}+|v|^{2(1-\theta)}\right) d x .
$$


This is possible only if $v \equiv 0$. A bounded solution should have an $\omega$-limit set, which is clearly impossible.

\section{b) Bounded domains.}

Compactly supported solutions in balls can be extended to larger bounded domains. We shall therefore consider exclusively solutions in balls.

The above parametrization can be used to fully determine the branches of radial solutions of the Dirichlet problem

$$
\left\{\begin{array}{l}
\Delta v+\mu v-|v|^{-2 \theta} v=0 \text { in } B \\
v=0 \text { on } \partial B
\end{array}\right.
$$

in a ball $B$ (which can be taken equal to the unit ball $B=B(0,1)$ without loss of generality). If we denote by $R_{k}(\lambda)$ the $k^{\text {th }}$ root of $u_{\lambda}$ (when it exists i.e. for $\left.k \leq N_{\lambda}(\infty)\right)$, then $R_{k}(\lambda)$ is increasing with respect to $k \geq 1$. Moreover

$$
\forall k \geq 1, \quad k \leq N_{\lambda} \quad \Longrightarrow \quad R_{k}(\lambda) \geq \inf _{\lambda \in(0,+\infty) \backslash A_{0}} R_{1}(\lambda)>0
$$

since $R_{1}(\lambda)>S(\lambda)>\sqrt{d}$ for $\lambda>0$ large enough, according to estimate (6). Branches of solutions of (18) whose support is exactly $B$ (see Fig. 5, Section 4) are then all given by

$$
\mu=\left(R_{k}(\lambda)\right)^{2} \quad \text { and } \quad v(x)=\left(R_{k}(\lambda)\right)^{-1 / \theta} u_{\lambda}\left(R_{k}(\lambda) x\right)
$$

where $u_{\lambda}$ is the solution of (3) with $\lambda>0$ (up to a change of sign: see Remark 1 for $\lambda<0$ ), provided

$$
\lambda \in B_{k}:=\bigcup_{j \geq k}\left(A_{j} \cup I_{j}\right) \underset{\varsubsetneqq}{\subsetneq}(0,+\infty) .
$$

Note that in the nonuniqueness cases, we can always choose $u_{\lambda}$ as the unique compactly supported solution. The classification of radial solutions is then completed if we take into account all radial compactly supported solutions whose support is strictly contained in $B$, corresponding to $\lambda \in I_{k}$, and given by

$$
\mu>\left(R_{k}(\lambda)\right)^{2} \quad \text { and } \quad v(x)=\mu^{-\frac{1}{2 \theta}} u_{\lambda}(\mu x) .
$$

Because of (7), when $\lambda$ goes to $+\infty$, the solution $u_{\lambda}$ of (2) belongs to $B_{k}$. We may therefore consider $\lim _{\lambda \rightarrow+\infty} R_{k}(\lambda)$, which is finite because of the asymptotic linear growth of $f$. Note that an upper bound of $R_{k}(\lambda)$ easily follows from Proposition 1:

$$
k=N_{\lambda}\left(R_{k}(\lambda)\right) \geq \frac{\theta(1-\alpha)}{\pi}\left(R_{k}(\lambda)-r_{\alpha}\right) \quad \forall \alpha \in(0,1)
$$

with $r_{\alpha}=\frac{d-1}{2 \alpha \theta}$, which means

$$
R_{k}(\lambda) \leq \inf _{\alpha \in(0,1)}\left(\frac{\pi k}{\theta(1-\alpha)}+\frac{d-1}{2 \alpha \theta}\right) .
$$

Because of (19) and (22), for any $k \in \mathbb{N}$, there exist two positive constants $\mu_{-}^{k}$ and $\mu_{+}^{k}$ such that all solutions of (17) with $k$ nodes and support equal to $B$ 
are such that $\mu_{-}^{k} \leq \mu \leq \mu_{+}^{k}$. These solutions form a branch parametrized by $\lambda$, which has a single component if and only if $B_{k}$ is made of only one interval. If $B_{k}$ is an interval for any $k \geq 1, J_{k}=A_{k} \cup I_{k}$ is a sequence of open to the left and closed to the right intervals. If the property is true for any $k \in \mathbb{N}, J_{k}$ is a sequence of ordered intervals:

$$
\forall k \in \mathbb{N} \quad \max J_{k}=\inf J_{k+1} .
$$

We may also notice that (7) means that the number of nodes of radial solutions $u$ of (2) (in the whole space $\mathbb{R}^{d}$ ) increases at least like $\|u\|_{L^{\infty}\left(\mathbb{R}^{d}\right)}^{1 /(d-1)}$, but an upper bound seems more difficult to obtain.

c) Non-radial solutions and more general nonlinearities.

The situation is much more delicate for non radial solutions which may change sign. However non-negative (up to a change of sign) solutions in $H^{1}\left(\mathbb{R}^{d}\right)$ have been completely classified in [11]: the support of any such solution is a finite union of disjoint balls. Each ball is of radius $\mu^{-1 / 2} R_{1}(\lambda)$ for $\lambda \in I_{1}$. According to [26] (also see references therein for earlier results), $I_{1}$ is reduced to a single point: all balls have the same radius. On each ball, the (unique up to a translation) solution is radial and strictly decreasing. This is of course possible because Equation (1) is autonomous so that any translate of a solution is still a solution. Such solutions are said to be locally radially symmetric and it has been proved in $[7,8,14$, 15] that this is the only possible way of breaking the radial symmetry of nonnegative solutions when the nonlinearity is continuous but not Lipschitz - here at $u=0$. It is very likely that there are other ways of breaking the symmetry of sign changing solutions, but this is for the moment an open question.

The extension of the results of this paper to general nonlinearities $f(u)$ which have an asymptotically linear growth as $u \rightarrow+\infty$ and are sublinear negative close to 0 is straightforward. The condition that $s \mapsto(-F(s))^{-1 / 2}$ is integrable at $s=0_{+}$is necessary and sufficient to guarantee the existence of solutions with compact support (see for instance [27]). It is also possible to generalize our approach to continuous but not locally Lipschitz nonlinearities. Uniqueness can be lost at each value of $u$ corresponding to a root of $f$, for which $f$ is not Lipschitz and satisfies a condition which generalizes the above condition (see [14, 15] for related results). The crucial argument for uniqueness is a local inversion which was introduced in [25] and has been used in the proof of Proposition 7.

\section{Figures}

Here are plotted results of numerical computations which illustrate Proposition 6 and the trapping region of Proposition 3 , for $d=3$ and $\theta=\frac{1}{4}$. The non-negative solution with compact support corresponds to $\lambda=4.86728 \ldots$ The solution with compact support and one node (i.e. changing sign once) is given by $\lambda=10.16313 \ldots$ A short discussion of branches of $H^{1}$ solutions on $\mathbb{R}^{d}$ and on the ball is also provided.

We numerically observe that $H^{1}\left(\mathbb{R}^{d}\right)$ solutions are ordered by their number of nodes, but we did not prove such a result. Uniqueness (up to a change of sign) of solutions with a given number of nodes is not proved and we cannot even exclude the existence of non discrete sets of solutions. 


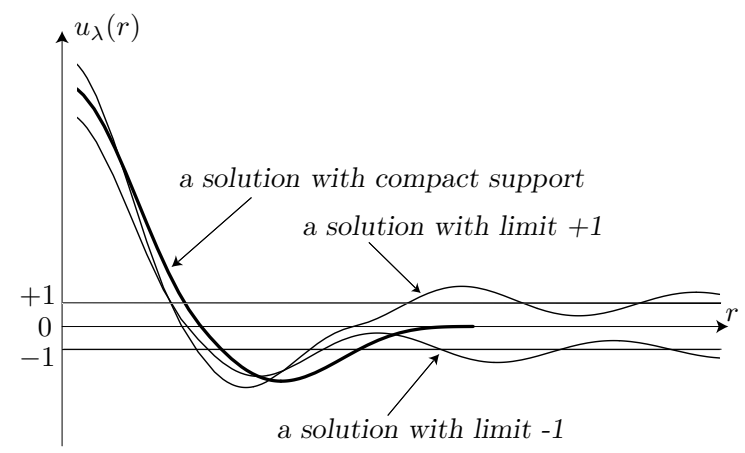

Fig. 1. For $d>1$, depending on the value of $u_{\lambda}(0)=\lambda$, the limit of $u_{\lambda}(r)$ as $r \rightarrow+\infty$ is -1 , 0 or +1 . In case it is 0 , the solution has a compact support.

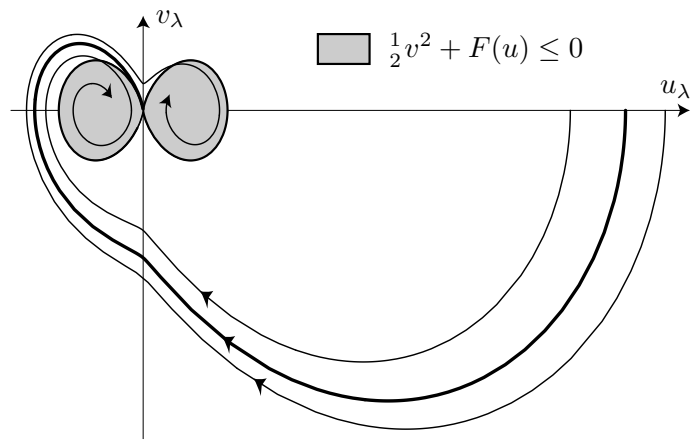

Fig. 2. For $d>1$, the energy of any solution $u_{\lambda}$ is decaying. Once it becomes negative, the number of its roots does not increase any more.

\section{References}

1. M. Balabane, T. Cazenave, A. Douady \& F. Merle, Existence d'états excités pour une équation de Dirac non linéaire [Existence of excited states for a nonlinear Dirac equation], C. R. Acad. Sci. Paris Sér. I Math. 306 (1988), no. 3, 117-120.

2. M. Balabane, T. Cazenave, A. Douady \& F. Merle, Existence of excited states for a nonlinear Dirac field, Comm. Math. Phys. 119 (1988), no. 1, 153-176.

3. M. Balabane, T. Cazenave \& L. Vázquez, Existence of standing waves for Dirac fields with singular nonlinearities, Comm. Math. Phys. 133 (1990), no. 1, 53-74.

4. R.D. Benguria, J. Dolbeault, M.J. Esteban, Classification of the solutions of semilinear elliptic problems in a ball, J. Differential Equations 167 (2000) 438-466.

5. P. Benilan, H. Brezis \& M.G. Crandall, A semilinear equation in $L^{1}\left(\mathbb{R}^{N}\right)$, Ann. Scuola Norm. Sup. Pisa Cl. Sci. (4) 2 (1975), no. 4, 523-555.

6. H. Berestycki \& P.-L. Lions, Nonlinear scalar field equations. II. Existence of infinitely many solutions, Arch. Rational Mech. Anal. 82 (1983), no. 4, 347-375.

7. F. Brock, Continuous Steiner symmetrization, Math. Nachrichten 172 (1995) 25-48.

8. F. Brock, Continuous rearrangements and symmetry of solutions of elliptic problems, Preprint (1999). 


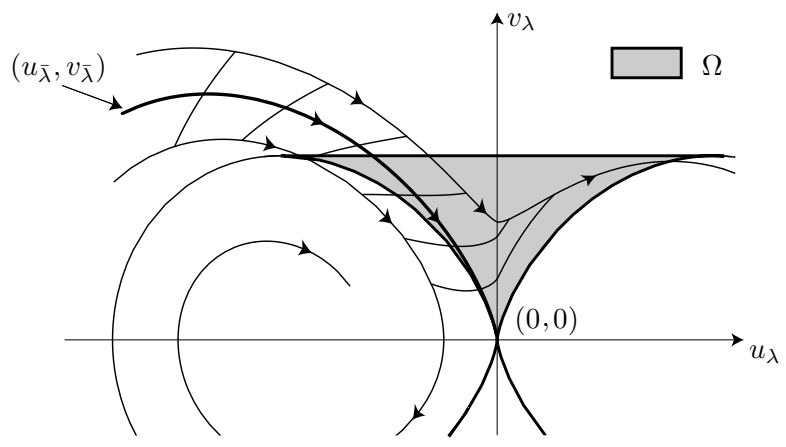

Fig. 3. The set $\Omega=\left\{(u, v): E=\frac{1}{2} v^{2}+F(u)>0,0<v<\sqrt{\frac{\theta}{1-\theta}}\right\}$ is a trapping region for $d>1$. A small interval $(\bar{\lambda}-\epsilon, \bar{\lambda}+\epsilon)$ of initial data is transported continuously by the flow as long as no solution corresponding to these initial data hits $(0,0)$, i.e. for $r>0$ not too large, but breaks if the interval contains a compactly supported solution for $r$ larger than the radius of the smallest support of a compactly supported solution with initial datum in $(\bar{\lambda}-\epsilon, \bar{\lambda}+\epsilon)$. Curves which are transverse to the flow represent the image of $(\bar{\lambda}-\epsilon, \bar{\lambda}+\epsilon)$ for various $r$ (less than the size of the support of $u_{\bar{\lambda}}$, which is compact).

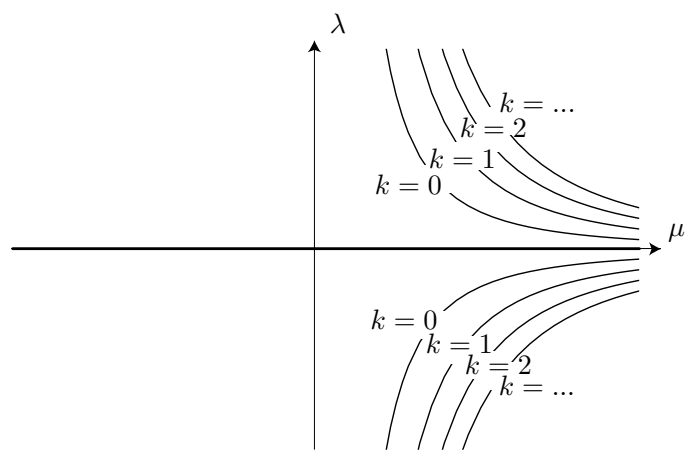

Fig. 4. Branches of radial solutions in $H^{1}\left(\mathbb{R}^{d}\right)$ of $\Delta v+\mu v-|v|^{-2 \theta} v=0$ with $k$ nodes. Here $\lambda=\|v\|_{L^{\infty}\left(\mathbb{R}^{d}\right)}$. The constant function 0 is a solution for any $\mu$, and there are no other radial solutions for $\mu \leq 0$. Note that for $d=3$ and $\theta=1 / 4$, we numerically observe branches of $H^{1}$ solutions which are ordered by their number of nodes, but we did not prove such a result and cannot even exclude the existence of larger sets of solutions.

9. T. Cazenave, F. Dickstein \& M. Escobedo, A semilinear heat equation with concave-convex nonlinearity, Rend. Mat. Roma, Serie VII 19, 211-242.

10. E. A. Coddington \& N. Levinson. Theory of Ordinary Differential Equations. McGrawHill, 1955.

11. C. Cortazár, M. Elgueta \& P. Felmer, On a semilinear elliptic problem in $\mathbb{R}^{N}$ with a non-Lipschitzian non-linearity, Advances in Differential Equations 1 no. 2 (1996) 199-218.

12. E. Deumens \& H. Warchall, Explicit construction of all spherically symmetric solitary waves for a nonlinear wave equation in multiple dimensions, Nonlinear Anal. 12 (1988), no. 4, 419-447.

13. J.I. Díaz, Nonlinear partial differential equations and free boundaries. Vol. I. Elliptic equations. Research Notes in Mathematics, 106. Pitman (Advanced Publishing Program), Boston, Mass.-London, 1985.

14. J. Dolbeault \& P. Felmer, Symétrie pour des équations semi-linéaires elliptiques (Symmetry of the solutions of semilinear elliptic equations), C. R. Acad. Sci., Paris, Sér. I, Math. 


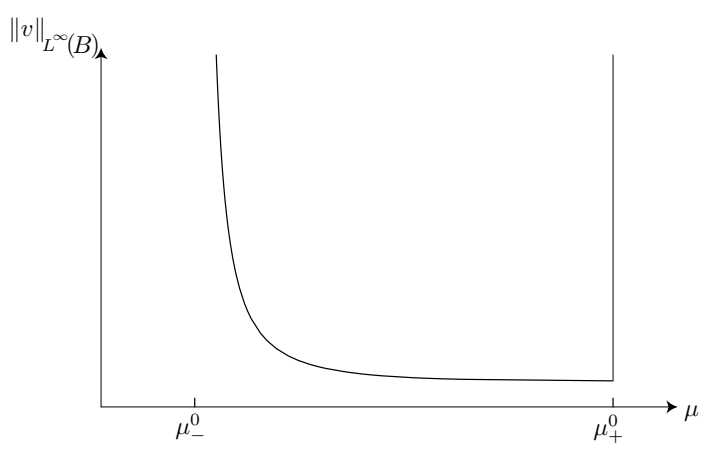

Fig. 5. The set of radial non-negative $(k=0)$ solutions of the Dirichlet problem (18) in the unit ball $B \subset \mathbb{R}^{3}$, is made of two branches. The vertical line at $\mu=\mu_{+}^{0}=53.9418 \ldots$ corresponds to radial solutions with support in a ball strictly contained in $B$, given by (21) for $\lambda=4.86728 \ldots$ The other branch corresponds to positive solutions in $B$ and is parametrized according to $(20)$ by $\lambda \in(4.86728 \ldots,+\infty)$. This branch has an asymptote at $\mu=\mu_{-}^{0}$ which is numerically found at $\pi^{2}$. Sets of radial solutions with $k \geq 1$ have a similar structure with $\mu_{-}^{k}=((2 k+1) \pi)^{2}$, which are the eigenvalues of $-\Delta$ in $B \subset \mathbb{R}^{3}$ with zero Dirichlet boundary conditions.

329 (1999), No. 8, 677-682.

15. J. Dolbeault \& P. Felmer, Symmetry and monotonicity properties for positive solutions of semi-linear elliptic pde's, Commun. Partial Differ. Equations 25, (2000) No.5-6, 1153-1169.

16. B. Franchi, E. Lanconelli, J. Serrin, Existence and uniqueness of non-negative solutions of quasilinear equations in $\mathbb{R}^{n}$, Adv. Math. 118 (1996), no. 2, 177-243.

17. M. Grillakis, Existence of nodal solutions of semilinear equations in $\mathbb{R}^{N}$, J. Differential Equations 85 (1990), no. 2, 367-400.

18. J.A. Iaia, H.A. Warchall \& F.B. Weissler, Localized solutions of sublinear elliptic equations: loitering at the hilltop, Rocky Mountain J. Math. 27 (1997), no. 4, 1131-1157.

19. C. Jones \& T. Küpper, On the infinitely many solutions of a semilinear elliptic equation, SIAM J. Math. Anal. 17 (1986), no. 4, 803-835.

20. R. Kajikiya, Nodal solutions of superlinear elliptic equations in symmetric domains, Adv. Math. Sci. Appl. 3 (1993/94), Special Issue, 219-266.

21. R. Kajikiya, Norm estimates for radially symmetric solutions of semilinear elliptic equations, Trans. Amer. Math. Soc. 347 (1995), no. 4, 1163-1199.

22. H.G. Kaper, M.K. Kwong \& Y. Li, Symmetry results for reaction-diffusion equations, Differential Integral Equations 6 (1993), no. 5, 1045-1056.

23. K. McLeod, W.C. Troy \& F. B. Weissler, Radial solutions of $\Delta u+f(u)=0$ with prescribed numbers of zeros, J. Differential Equations 83 (1990), no. 2, 368-378.

24. Y. Naito, Bounded solutions with prescribed numbers of zeros for the Emden-Fowler differential equation, Hiroshima Math. J. 24 (1994), no. 1, 177-220.

25. L.A. Peletier \& J. Serrin, Uniqueness of positive solutions of semilinear equations in $R^{n}$, Arch. Rational Mech. Anal. 81 (1983), no. 2, 181-197.

26. J. Serrin \& M. Tang, Uniqueness of ground states for quasilinear elliptic equations, Indiana Univ. Math. J. 49 (2000), no. 3, 897-923.

27. J. Serrin \& H. Zou, Symmetry of ground states of quasilinear elliptic equations, Arch. Rational Mech. Anal. 148 (1999), 265-290.

28. W.A. Strauss, Existence of solitary waves in higher dimensions, Comm. Math. Phys. 55 (1977), no. $2,149-162$. 